\title{
Hemothorax Due to Rupture of a Benign Thymoma
}

John L. CaPlin, M.B., M.R.C.P., Richard W. Gullan, M.B., B.S., Duncan S. Dymond, M.D., M.R.G.P., Stephen M.O. Bradeey, M.B., F.R.C.S.,

Ian M. Hill, M.S., F.R.C.S., and Seamus O. Banim, M.B., F.R.C.P.

\section{Summary}

Non-traumatic hemothorax is rare and in the case we report was due to rupture of a benign thymoma. The clinical course of the patient suggested rapid intrathoracic bleeding and emergency surgery was required to make the correct diagnosis.

\section{Additional Indexing Words :}

Thymic tumor Rapid intrathoracic bleeding

ON-traumatic hemothorax, with blood but no air in the pleural space, 1 is a rare but well recognized condition. Causes include bleeding tumors of the lung or pleura, dissecting aneurysm of the thoracic aorta, endometriosis, various bleeding disorders and anticoagulant therapy. ${ }^{11,2)}$ The amount and rapidity of hemorrhage depends upon the etiology, and the diagnosis is often made only at surgery, which may be an emergency procedure if the bleeding persists or is severe. We report a case where rupture of a benign thymoma caused a hemothorax which required urgent surgery to control the recurrent bleeding.

\section{Case Report}

A 51-ycar-old Nigerian man, who had been resident in the United Kingdom for 22 years, presented with a 2-day history of left sided chest pain and dyspnea. The pain was constant, non-pleuritic and radiated to the left shoulder. There was no sputum or hemoptysis. He had been well until the onset of the pain and there was no past history of cardiac or pulmonary disease or tuberculosis. Examination showed him to be apyrexial with tenderness over the left side of his chest and the signs of a large left pleural effusion with

From the Departments of Cardiology and Cardiothoracic Surgery, St. Bartholomew's Hospital, West Smithfield, London, EC1A 7BE, England.

Address for reprint: Dr. J.L. Caplin, Cardiac Department, St. Bartholomcw's Hospital, West Smithfield, London, ECiA 7BE, England.

Received for publication January 9, 1984. 


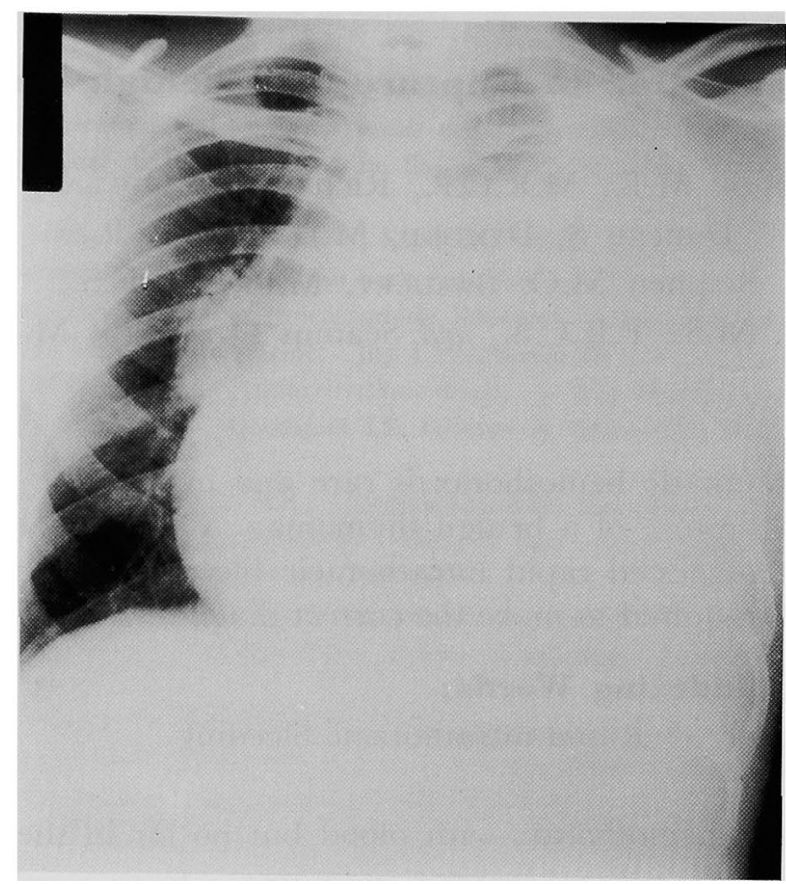

Fig. 1. PA chest X-ray.

mediastinal shift to the right.

Electrocardiogram showed a sinus tachycardia with otherwise normal complexes and a chest radiograph (Fig. 1) showed a large left pleural effusion with shift of the mediastinum to the right.

Hemoglobin was $10.3 \mathrm{Gm} / \mathrm{d} l$ and the white blood cell count was 22.0 $\times 10^{9} / l\left(22,000 / \mathrm{mm}^{3}\right)$. Approximately 1 liter of the effusion was aspirated and the fluid was heavily blood stained, with a hemoglobin concentration of $5.0 \mathrm{Gm} / \mathrm{d} l$. Within 12 hours the effusion had reaccumulated and the patient had become hypotensive with a tachycardia and the signs of mediastinal shift to the right. An emergency left postero-lateral thoracotomy was performed and revealed a ruptured thymic tumor in the anterior mediastinum with over 2 liters of blood in the left pleural space. The tumor was completely removed and was histologically a benign, epithelium predominant, thymoma. The post-operative course was uneventful. A routine chest X-ray that had been performed 5 years previously, was subsequently available for review. Although, at the time, it had been reported as normal, the review revealed a rounded shadow in the anterior, upper mediastinum. 


\section{Discussion}

Non-traumatic hemothorax is a rare clinical problem with a limited number of known causes. This report presents a further hitherto unreported cause and illustrates that the cause may be present for some years without problems, but that massive bleeding may occur suddenly, without warning, and endanger the patient's life.

Our patient presumably had a benign thymic tumor for many years without any compressive symptoms or non-localized complications such as myasthenia gravis or pure red blood cell aplasias. ${ }^{3)}$ He then presented with a short history of chest and shoulder pain and with the signs of a pleural effusion. Pleurocentesis showed this to be heavily blood-stained confirming the diagnosis of hemothorax. Within 12 hours blood had reaccumulated and the patient's clinical state necessitated emergency surgery.

Urgent or emergency surgery is often required especially if the bleeding is rapid and often definitive diagnosis is only obtained at operation. ${ }^{21}$ However where hemothorax is secondary to a small pneumothorax, chest drainage and blood transfusion have been used to treat patients, with a resulting low recurrence rate. ${ }^{4)}$

Rupture of a benign thymoma is unusual, but rupture of a thymic teratoma resulting in a left hemothorax has been reported in a 14-year-old boy and presented in a similar manner to this case. It too required emergency surgery to make the diagnosis. ${ }^{5}$

In conclusion, thymic tumors should be considered in patients presenting with a spontaneous hemothorax and bleeding from ruptured thymic tumors may be severe and require urgent surgery.

\section{ReFERENCES}

1. Emerson P: Haemothorax. in Thoracic Medicine, ed by Emerson P, Butterworths, London, p 629-30, 1981

2. Fishman A: The pleural space. in Pulmonary Disease and Disorders, ed by Fishman A, McGraw Hill, New York, p 1368-70, 1980

3. Loon G, Schiby G, Milo S: Lesion of the thymus. Israel J Med Sci 16: 433, 1980

4. Van Way C: Massive spontancous hacmothorax. Arch Surg 114: 1443, 1979

5. Botha R, Brown S, Pursel S, Chandel M: Thymic teratoma. Spontaneous rupture into the pleural space. Postgrad Med 56: 193, 1974 